\title{
Fine Structure of Neutral Excitons in Single GaAlAs Quantum Dots
}

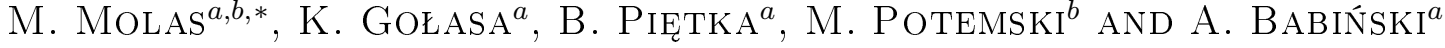 \\ ${ }^{a}$ Institute of Experimental Physics, Faculty of Physics, University of Warsaw \\ Hoża 69, PL-00-681 Warsaw, Poland \\ ${ }^{b}$ Laboratoire National des Champs Magnétiques Intenses, CNRS-UJF-UPS-INSA \\ 25, av. des Martyrs, 38042 Grenoble, France
}

\begin{abstract}
Optical anisotropy of neutral excitons in GaAlAs/AlAs quantum dots is investigated. Low-temperature polarization-sensitive photoluminescence measurements of single quantum dots are performed. It is found that neutral excitons $(\mathrm{X})$ in the quantum dots exhibit a fine structure splitting. The fine structure splitting ranges from $10 \mu \mathrm{eV}$ to $100 \mu \mathrm{eV}$ and correlates with the $\mathrm{X}$ energy. The polarization axis of the fine structure splitting is well oriented along [110] crystallographic direction of a substrate. The orientation is attributed to the elongation of GaAlAs/AlAs quantum dots in the [110] direction of the substrate.
\end{abstract}

PACS: 78.55.Cr, 78.67.Hc

\section{Introduction}

Semiconductor quantum dots (QDs) keep attracting much interest due to their applications in quantum information processing and communication technologies. Therefore the detailed knowledge of symmetries and level-structure of these artificial atoms in the context of the QDs structural properties is essential. Information on the symmetry of the QDs confining potential can be e.g. acquired from investigation of a neutral exciton (X) in a QD. The neutral exciton consists of an electron-hole pair confined in the dot. Due to the symmetric electron-hole exchange interaction the exciton splits into the dark and bright configuration. If the confining potential symmetry is lowered, the latter states split [1] due to the anisotropic long-range electron-hole exchange interaction [2,3]. The fine structure splitting (FSS) of a neutral exciton is a crucial parameter from the point of view of entangled photon generation [1]. In this paper we study the optical properties (FSS and polarization orientation) of GaAlAs/ AlAs QDs in order to get an insight into the microscopic structure of the QDs.

\section{Experimental procedure}

The sample under study consists of a type II GaAs/ AlAs double quantum well structure $[4,5]$ (see inset in Fig. 1). The appropriate choice of both GaAs and AlAs layer thickness allows to observe not only the direct recombination of excitons $\left(\Gamma_{\mathrm{GaAs}}-\Gamma_{\mathrm{GaAs}}\right)$ in the

\footnotetext{
* corresponding author; e-mail: maciej.molas@fuw.edu.pl
}

GaAs quantum well, but also the indirect recombination $\left(X_{\mathrm{AlAs}}^{Z}-\Gamma_{\mathrm{GaAs}}, X_{\mathrm{AlAs}}^{X Y}-\Gamma_{\mathrm{GaAs}}\right)$ in the GaAs/AlAs bilayer. Additional series of sharp peaks can be also observed in the macro-photoluminescence (macro-PL) spectrum of the structure in the $1.56-1.68 \mathrm{eV}$ energy range (Fig. 1).

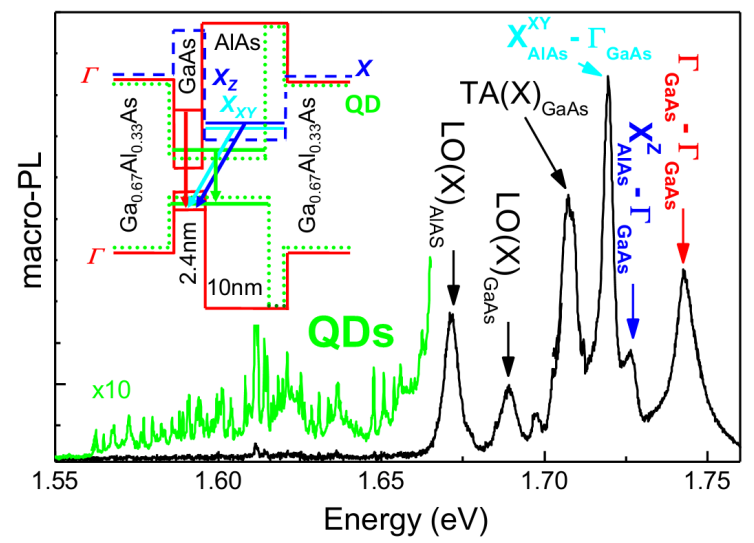

Fig. 1. Macro-photoluminescence spectrum and scheme of the energy distribution (inset) of the studied structure.

Previous studies showed that the emission lines are due to natural QDs, which form during the sample growth at interface imperfections. Inside the QD, the $\mathrm{GaAs} / \mathrm{AlAs}$ bilayer is replaced by $\mathrm{Ga}_{1-x} \mathrm{Al}_{x} \mathrm{As}$ material $(x<0.33)$. This lowers the potential resulting in the QDs formation. The QDs density as low as $10^{6} \mathrm{~cm}^{-2}$ was previously determined in the sample [6]. Such a low QD density allows to excite individual QDs without using metallic masks or 
spectral filtering. The $\mathrm{Ar}^{+}$laser $(\lambda=488 \mathrm{~nm})$ was used for micro-photoluminescence (micro-PL) excitation. The sample was placed on a cold finger of a continuous flow cryostat at liquid helium temperature $(T=4.2 \mathrm{~K})$. The excitation light was delivered and the PL was collected via a microscope objective (spot size around $1 \mu \mathrm{m}$ ), dispersed by using a $1 \mathrm{~m}$ monochromator and detected by a nitrogen cooled CCD camera [7]. Polarization-resolved experiments were implemented using a motorized rotating half-wave plate combined with a fixed linear polariser in front of the spectrometer in order to avoid detection artefacts related to the anisotropic response function of the setup.

\section{Results and discussion}

A typical PL spectrum of a single GaAlAs QD is shown in Fig. 2a. At the lowest excitation power only one emission line is observed, which is attributed to an $\mathrm{X}$ in a QD. With increasing power, next two emission lines $\mathrm{X}^{-}$, and $\mathrm{X}^{2-}$ emerge at lower energy. The emission lines are tentatively attributed to multicharged excitons. Similar detection of several excitonic lines due to different charge states of a dot is often reported in III-V QDs [8-10]. The excitation power dependence of lines $\mathrm{X}, \mathrm{X}^{-}$, and $\mathrm{X}^{2-}$ is presented in Fig. 2b. Intensities of emission lines increase with increasing excitation power (around linearly for $\mathrm{X}$, quadratically for $\mathrm{X}^{-}$and $\mathrm{X}^{2-}$ ). At the highest excitation power a broad emission band, marked as MX in Fig. 2a emerges at lower energy range, which is attributed to a multiexcitonic emission from a single dot.

In order to identify the observed emission lines polarization-sensitive micro-PL measurements were performed. For the determination of the FSS, we first fit the different excitonic lines with Gaussian curves as a function of the polarization angle. The polarization dependence of the observed features is shown in Fig. 2c. It can be seen that both $\mathrm{X}$ and $\mathrm{X}^{2-}$ lines comprise two components linearly polarized in perpendicular directions. Their polarization axes are identical within an experimental error and the respective FSS for $\mathrm{X}$ and for $\mathrm{X}^{2-}$ emission can be considered equal. The $\mathrm{X}^{-}$emission line is not polarized. Our results confirm the attribution of the $\mathrm{X}$ emission line to a neutral exciton and $\mathrm{X}^{-}$emission line to a charged exciton. Less straightforward is the identification of the $\mathrm{X}^{2-}$. We tentatively attribute the $\mathrm{X}^{2-}$ line to a doubly charged exciton, however more detailed studies confirming this statement are beyond the scope of this paper.

The FSS of a neutral exciton was measured in more details and the results are summarized in Fig. 3. The related X-emission energy ranges from $1.57 \mathrm{eV}$ to $1.69 \mathrm{eV}$, which suggests substantial dispersion of QDs size and Al composition inside dot and the barrier. The FSS energy of neutral excitons confined in the QDs is dispersed in a very broad range (from about $10 \mu \mathrm{eV}$ to $100 \mu \mathrm{eV}$ ).

In the III-V QDs the FSS usually decreases as a function of the $\mathrm{X}$ energy emission (the FSS is smaller for

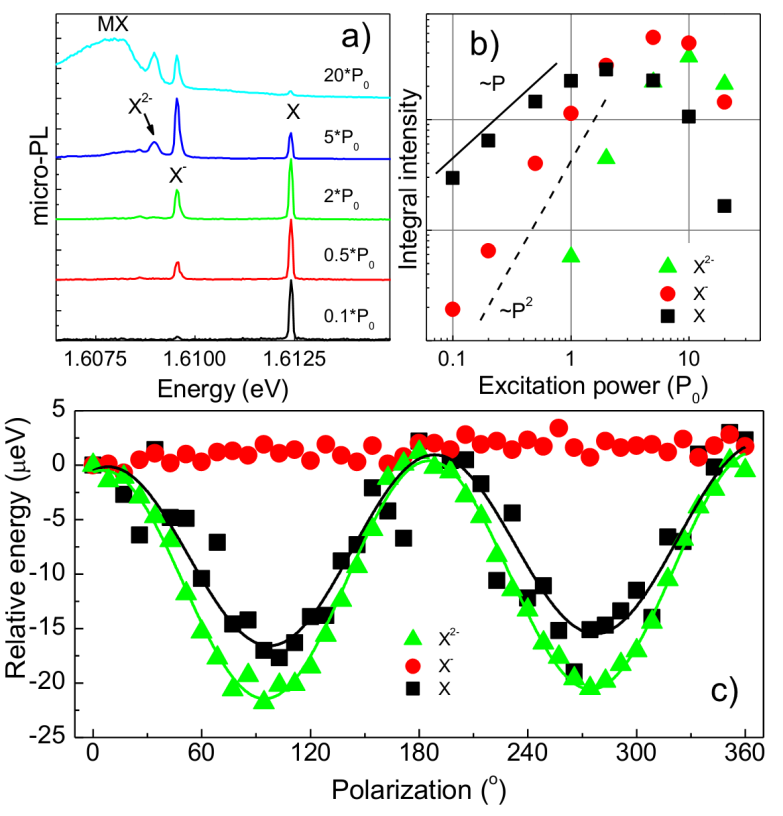

Fig. 2. (a) Luminescence spectrum of a single GaAlAs/AlAs QD excited with increasing excitation power density $\left(P_{0}=1 \mu \mathrm{W}\right)$. The three lines $\mathrm{X}, \mathrm{X}^{-}$, and $\mathrm{X}^{2-}$ are interpreted as neutral, charged, and doubly-charged exciton. (b) Power dependence of $\mathrm{X}, \mathrm{X}^{-}$, and $\mathrm{X}^{2-}$. Linear and quadratic behaviour of intensity versus excitation power is also shown as a guide to the eye. (c) Energy evolution of $\mathrm{X}, \mathrm{X}^{-}$, and $\mathrm{X}^{2-}$ with polarization axis (points). Fit is a cosine square function as evidenced by solid lines thus determining amplitude (the FSS).

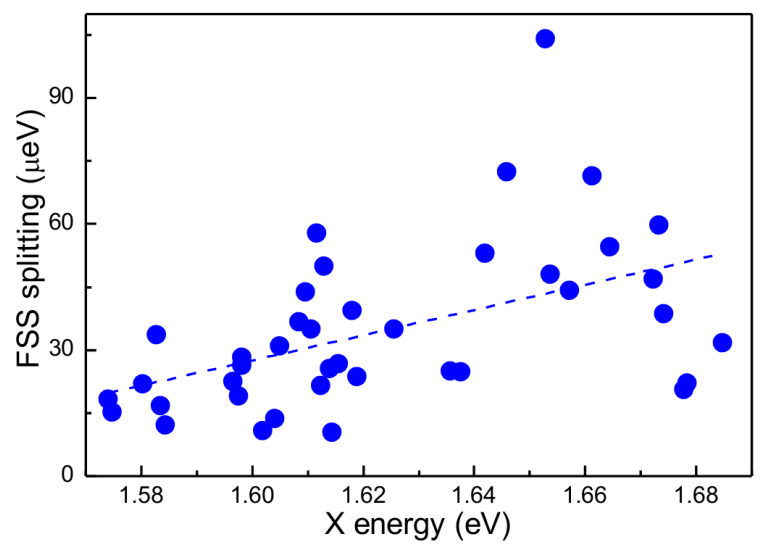

Fig. 3. The FSS of the GaAlAs/AlAs QDs emitting at different energies. Dashed line is a guide to the eye. Uncertainly of the FSS splitting determination was estimated as a few $\mu \mathrm{eV}$.

larger dots). This is due to piezoelectric field as already discussed in Ref. [8]. The trend observed in the investigated structure is opposite, which can be related to the effect of QD elongation [11].

In order to get further information on the origin of the FSS in the investigated structure the energy dependence 


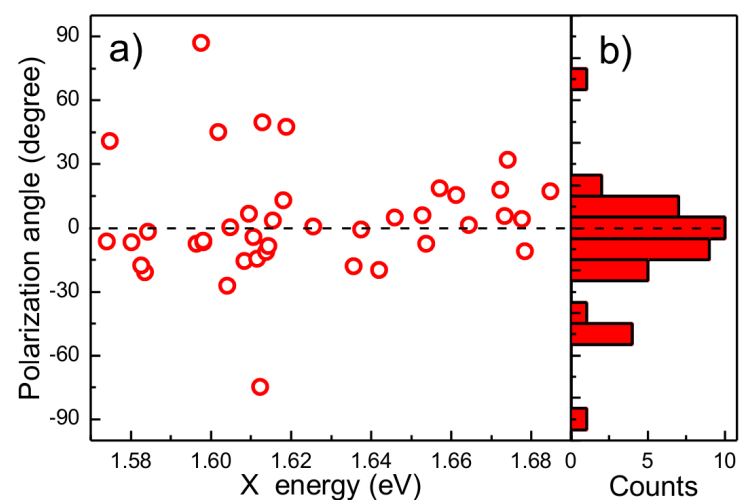

Fig. 4. (a) Polarization axis orientation with respect to the [110] axis for the investigated GaAlAs/AlAs dots. (b) Histogram of the dot distribution along polarization axis.

of the X-polarization axis was also studied, which Fig. 4 illustrates.

It was observed that in most of investigated QDs the optical anisotropy direction (defined as the direction of the lower energy component of the $\mathrm{X}$ emission line) coincided with the crystallographic [110] direction. Orientation of the $\mathrm{X}$ polarization axis along [110] crystallographic axis of the substrate suggests that the elongation of the dot along the axis has the main effect on the QDs optical anisotropy. The elongation most likely results from different diffusion rates of $\mathrm{Al}$ atoms along the direction parallel and perpendicular to atomic steps of the GaAlAs/AlAs interface.

\section{Conclusions}

We have reported on detailed measurements of the FSS of single GaAlAs/AlAs QDs. We have found that the FSS ranges from $10 \mu \mathrm{eV}$ to $100 \mu \mathrm{eV}$ and it seems to correlate with the neutral exciton emission energy. The polarization axis of the FSS is along the [110] crystallographic direction of the substrate. It has been observed that the optical anisotropy of the investigated dots is related to the elongation of the GaAlAs/AlAs due to preferential diffusion of the $\mathrm{Al}$ atoms along the atomic steps at the GaAlAs/AlAs interface.

\section{Acknowledgments}

This work was partially supported by the Foundation for Polish Science International Ph.D. Projects Programme co-financed by the EU European Regional Development Fund.

\section{References}

[1] O. Benson, C. Santori, M. Pelton, Y. Yamamoto, Phys. Rev. Lett. 84, 2513 (2000).

[2] D. Gammon, E.S. Snow, B.V. Shanabrook, D.S. Katzer, D. Park, Phys. Rev. Lett. 76, 3005 (1996).

[3] L. Besombes, K. Kheng, D. Martrou, Phys. Rev. Lett. 85, 425 (2000).

[4] A. Wysmołek, B. Chwalisz, M. Potemski, R. Stępniewski, A. Babiński, S. Raymond, Acta Phys. Pol. A 106, 367 (2004).

[5] A. Trüby, M. Potemski, R. Planel, Solid-State Electron. 40, 139 (1996).

[6] B. Piętka, Ph.D. Thesis, Joseph Fourier University Grenoble I, University of Warsaw - Warsaw 2002.

[7] M. Molas, K. Gołasa, K. Kuldová, J. Borysiuk, A. Babiński, J. Lapointe, Z.R. Wasilewski, J. Appl. Phys. 111, 033510 (2012).

[8] T. Belhadj, T. Amand, A. Kunold, C.-M. Simon, T. Kuroda, M. Abbarchi, T. Mano, K. Sakoda, S. Kunz, X. Marie, B. Urbaszek, Appl. Phys. Lett. 97, 051111 (2010).

[9] M. Abbarchi, C.A. Mastrandrea, T. Kuroda, T. Mano, K. Sakoda, N. Koguchi, S. Sanguinetti, A. Vinattieri, M. Gurioli, Phys. Rev. B 78, 125321 (2008).

[10] A. Babinski, G. Ortner, S. Raymond, M. Potemski, M. Bayer, W. Sheng, P. Hawrylak, Z. Wasilewski, S. Fafard, A. Forchel, Phys. Rev. B 74, 075310 (2006).

[11] R. Seguin, A. Schliwa, S. Rodt, K. Pötschke, U.W. Pohl, D. Bimberg, Phys. Rev. Lett. 95, 257402 (2005). 